\title{
Parallel Synthesis of Unsymmetrical trans-Stilbenes
}

\author{
Chul-Hee Cho and Kwangyong Park*
}

\author{
School of Chemical Engineering \& Materials Science, Chung-Ang Chivensitv. Seoul 156-756, Korea. "E-mail: kyparkiácau ac.kr \\ Received March 23, 2007
}

\begin{abstract}
New unsymmetrical trans-stilbenes have been prepared by the sequential coupling reactions of bromobenzenesulfonate with formylarylboronic acids, benzylphosphonates and arylmagnesium bromides and characterized. The nickel-catalyzed reactions of stilbenesulfonates with aryl Grighard reagents produced the corresponding stilbenes via the nucleophilic aromatic substitution of the neopenty loxysulfonyl group by aryl nucleophiles. The great chemoselectivity of the alky loxysulfonyl group allows the stepwise construction of unsy mmetrical trans-stilbenes possessing terphenyl moieties. This procedure appears to be a promising and conceptually straightforward route for the parallel synthesis of various unsynmetrical stilbenes as well as other highly conjugated hydrocarbons
\end{abstract}

Key Words : Unsymmetrical trans-stilbenes. Stilbenesulfonates. Nickel-catalyst. Cross-coupling

\section{Introduction}

Stilbene and oligostilbene derivatives. which are frequently found in nature, have become of particular interest owing to their wide range of biological activities including anticancer, ${ }^{1}$ antifungal, ${ }^{2}$ antioxidant, ${ }^{3}$ anti-HIV ${ }^{4}$ and anti-inflammatory activities. Resveratrol. a hydroxylated $m$. $m$. compound isolated from various plant species, is especially attracting much attention due to its interesting phannacological properties. ${ }^{6}$ The stilbene skeleton has often been chosen as a central chromophore for the construction of bulky photoresponsive materials. ${ }^{7}$ Accordingly. the photochemical and photophysical properties of stilbene derivatives have been extensively studied as they are the prototype of the photochenical cis-frans isonerization of 1,2-disubstituted olefins. ${ }^{\text {S }}$ Stilbene derivatives are also attractive for technological applications in optical data storage ${ }^{9}$ and $\mathrm{NLO}^{1{ }^{13}}$

Stilbene derivatives have recently attracted great interest in the area of organic flexible displays. Since the first enitting conjugated poly ( $p$-phenylenevinylene) (PPV) was reported in $1990 .{ }^{11}$ PPV derivatives have been important as the emissive layer of organic light-emitting diodes (OLEDs). ${ }^{12}$ Stilbenes as well as the $\pi$-conjugated oligomers with alternating phenyl and vinyl units. short subunits of PPV conpounds, have been of wide interest in research to gain an understanding of the light emission mechanisms and to predict the influence of substitution on the emitted light. ${ }^{13}$ Also, stilbene derivatives are known to exhibit blue photoluminescence by themselves. ${ }^{1+}$ Aminostilbene compounds have been described as showing the fluorescence ${ }^{15}$ as well as the hole transport ${ }^{16}$ property. Stilbene dendrinters have recently attracted considerable attention as novel luninescent materials for applications in OLEDs. ${ }^{17}$

The most familiar synthetic pathways for unsymmetrical stilbenes $^{18}$ have adapted the Wittig ${ }^{19}$ and Honer-WadsworthEmmons (HWE) reactions ${ }^{2 i)}$ of substituted benzaldehydes with benzyl phosphonium ylides or phosphonates. Stilbenes are often elaborated from styrene derivatives via the Heck reaction. ${ }^{-1}$ Two sequential Heck reactions of haloarenes with ethene have been described for the preparation of stilbenes and PPVs. ${ }^{2}$ The combined use of the Heck and Wittig reactions ${ }^{23}$ or the Heck and $\mathrm{HWE}$ reactions ${ }^{24}$ has also been employed to construct the alternating phenyl and vinyl units. The Perkins reaction has also recently been applied for the synthesis of stilbene derivatives. ${ }^{25}$ Symmetrical stilbenes are often formed via dimerization reactions. Oxidative dimerization of methylarenes, ${ }^{26}$ eliminative dimerization of benzyl halides $^{27}$ and reductive dimerization of benzaldehydes ${ }^{28}$ have been reported for the efficient preparation of symmetrical stilbene compounds. However. there has been difficulty isolating the synthetic strategy appropriate for the rapid preparation of highly conjugated stilbenes possessing oligophenyl moieties.

In a program directed at the development of organic fluorescent materials. we have investigated an efficient synthetic pathway for the preparation of various highly conjugated stilbene and distyrylarylene derivatives. Recently, the neopentyloxysulfonyl groups. directly attached onto arenes, have been discovered to act as excellent leaving groups in the nickel-catalyzed reactions with aryl- and alkylmagnesium halides by our group. ${ }^{29}$ In this paper, our efforts in applying this new coupling reaction to the parallel synthesis of a unsynumetrical stilbene library are reported Unsymmetrical trans-stilbene derivatives 8 could be prepared by the nickel $(0)$-catalyzed cross-coupling reaction of trons-stilbenesulfonates 6 . which were prepared by the consecutive Suzuki-Miyaura coupling and HWE olefination of 4-bromobenzenesulfonate 2. with aryl Grignard reagents 7 (Scheme 1). All the stilbenesulfonates 6 and stilbenes 8 except 8 a prepared for this study, to our knowledge, have not been reported before. The results of this study are presented and discussed below:

\section{Results and Discussion}

Fonnylbipheny lsulfonates + were prepared by employing 


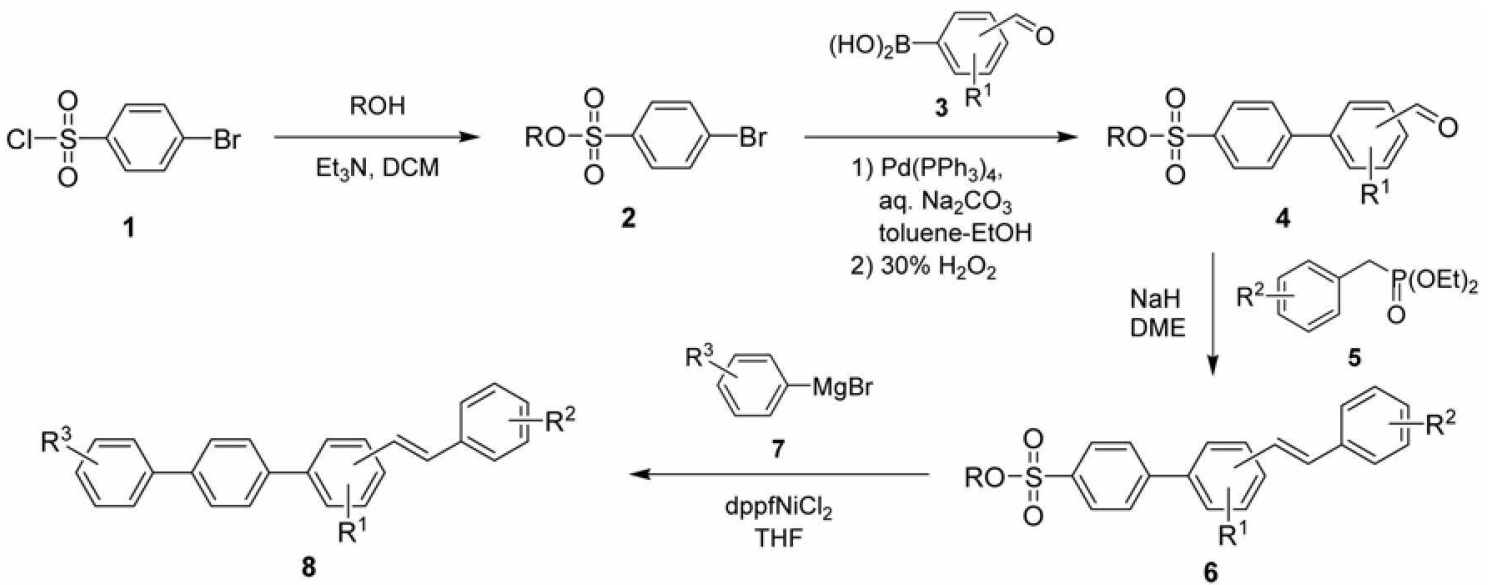

Scheme 1

a modification of literature procedure. ${ }^{31}$ The Suzuki-Miyaura coupling of bromobenzenesulfonate 2 . which was prepared by the reaction of neopentyl alcohol with 4-bromobenzene- sulfonyl chloride (1), with formylarylboronic acids 3 proceeded efficiently in the presence of a $\mathrm{Pd}\left(\mathrm{PPl}_{3}\right)_{4}$ catalyst. The neopentyl moiety, 2.2-dimethyl-3-phenyl-1-propyl. was

Table 1. Preparation of tran.s-stilbenesulfonates $6^{a}$

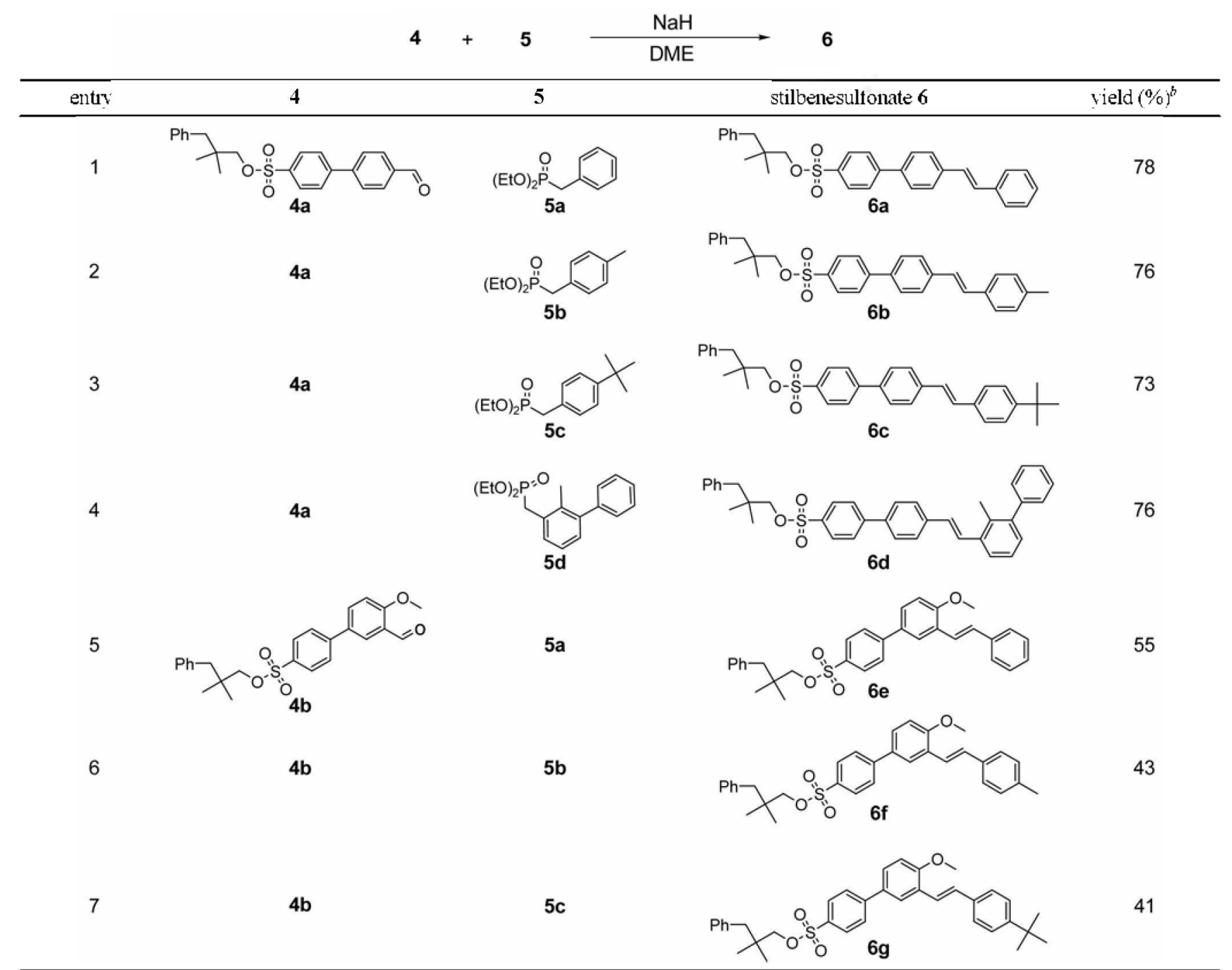

"Reactions of $4(2.80 \mathrm{mmol})$ with $5(3.08 \mathrm{mmol})$ were carried out in the presence of $\mathrm{NaH}(3.10 \mathrm{mmol})$ at the refluxing temperature of DME ( $30 \mathrm{~mL}$ ) for $1016 \mathrm{~h} .{ }^{6}$ Isolated yields based on 4. 
selected as the alkyl group of the sulfonates to avoid the competitive substitution or elimination of arenesulfonate anions in the subsequent reactions with strong nucleophiles. Both the coupling reactions of 4-formylphenylboronic acid (3a) and 3-formyl-4-methoxyphenylboronic acid (3b) proceeded well to produce the desired sulfonates ta and $\mathbf{t b}$. which were purified by simple recrystallization. in 68 and $71 \%$ yields respectively.

The formylbipheny lsulfonates $4 a$ and $\mathbf{4 b}$ reacted with four benzylphosphonates 5 to form stilbenesulfonates 6 by constructing the central vinyl moiety of the stilbene structure (Table 1), 4'-Fornyl-1, l'-biphenyl-4-sulfonate ta reacted with phosphonates 5a-5d to generate the stilbenesulfonates 6a-6d in good yields (entries 1-4). 3'-Formyl-4'-methoxyl, l'-biphenyl-4-sulfonate $\mathbf{4 b}$ was also reactive towards 5 a$5 \mathrm{c}$ rapidly generating the corresponding stilbenesulfonates $6 \mathrm{e}-6 \mathrm{~g}$ (entries 5-7). even though the yields of $6 \mathrm{e}-6 \mathrm{~g}$ were slightly lower than those of $6 \mathrm{a}-6 \mathrm{~d}$. All products, 6a-6g. were purified by recrystallization from either $i-\mathrm{PrOH}$ or $i-\mathrm{PrOH} n$ hexane (90:10)

All reactions showed great stereoselectivity in producing only the rrons-stilbenesulfonates within the limits of the NMR and GC detection. as expected from the literature. ${ }^{31}$ The coupling constants between the two vinylic protons of stilbenes 6 were $16 \cdot 17 \mathrm{~Hz}$, the typical coupling constants between the trans vicinal vinylic protons, in the ${ }^{1} \mathrm{H}-\mathrm{NMR}$ spectra.

Cross-coupling reactions of the stilbenesulfonates 6 with the aryl Grignard reagents 7 were carried out in the presence of a nickel $(0)$ catalyst, and the results of which are summarized in Table 2. Treatment of a mixture of sulfonates 6 and dppfNiCl: (5 mol\% based on 6 ) with the aryl Grignard reagents 7 in THF effectively produced unsymmetrical trons-stilbene derivatives $\mathbf{8}$ wia the nucleophilic aromatic substitution of the neopentyloxysulfonyl group by aryl nucleophiles. The optimized process discovered in our previous study. ${ }^{2 !+t}$ which used the $3+2$ equivalents of 7 to the sulfonates 6. was adapted. Although the stilbenesulfonates 6 dissolve poorly in THF at room temperature. they adequately dissolve by refluxing in THF to undergo the coupling reaction.

All the coupling reactions proceeded rapidly regardless of the stilbene structure and were finished within $12 \mathrm{~h}$. A faster reaction of the more conjugated arenesulfonates has been consistently' observed in previous work ${ }^{29}$ While no significant levels of byproducts originating from the sulfonates 6 were obtained a large amount of biphenyls derived by the dimerization of 7 were produced in these reactions. However. the biphenyls were easily removed by washing the crude solid products with methanol. Despite the efficient reaction. the poor solubility of the products in organic solvents seemed to decrease the final yields by making the purification difficult. Accordingly, the stilbenes that showed better solubility due to a greater number of alkyl or methoxy substituents were obtained in relatively higher yields (entries 5.6.8.9.12 and 13).

While all the ${ }^{~} \mathrm{H}$ NMR spectra of 8 were clear enough to
Table 2. Preparation of unsymmetrical trans-stilbenes $\mathbf{8}^{4}$

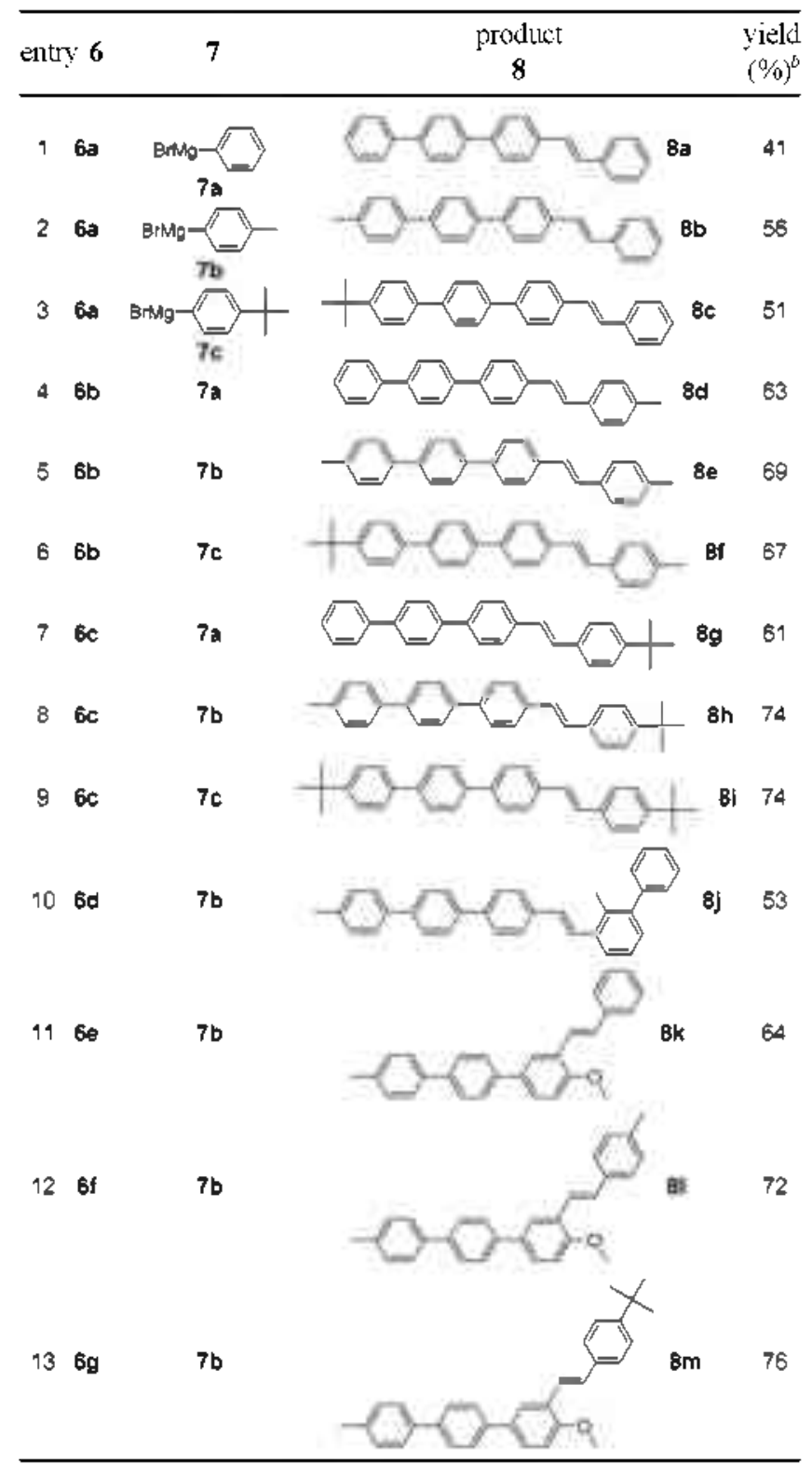

${ }^{a}$ Reactions of $6(0.100 \mathrm{mmol})$ with $7(0.50 \mathrm{mmol})$ were carried out at the refluxing temperature of THF $(20 \mathrm{~mL})$ using dpptNiCl $2(0.00500 \mathrm{mmol})$. Isolated vields based on 6 .

characterize their full chemical structures. it was extremely difficult to obtain good ${ }^{13} \mathrm{C}$ NMR spectra for most stilbenes 8 due to their poor solubility. Only $8 \mathbf{k}-8 \mathrm{~m}$, which show relatively better solubilities in $\mathrm{CDCl}_{3}$, allowed reasonably good ${ }^{13} \mathrm{C}$ NMR spectra to be obtained. A substituent of the phenyl ring. directly attached on the vinyl moiety. has an interesting influence on the chemical slift of the vinylic protons. Both of the vinylic protons of $8 \mathbf{a}$. $8 \mathbf{b}$ and $8 \mathbf{c}$. which contain no substituent on that phenyl group. are equivalent. and form a singlet in ${ }^{1} \mathrm{H}$ NMR spectra. However, each vinylic proton of the stilbenes possessing a methyl $(\mathbf{8 d}-\mathbf{8 f})$ or a tert-butyl $(\mathbf{8 g - 8 i )}$ group on the phenyl ring is different and generates a doublet in different positions of ${ }^{1} \mathrm{H}$ NMR spectra. A similar phenomenon was found in the ${ }^{\text {}} \mathrm{H}$ NMR spectra of the stilbenesulfonates 6 . The two vinylic protons 
of stilbenes $8 \mathrm{j}-8 \mathrm{~m}$ are also different, and form a pair of doublets. The coupling constants of those doublets in the ${ }^{1} \mathrm{H}$ NMR spectra of $8 \mathrm{~d}-8 \mathrm{~m}$ are $16-17 \mathrm{ppm}$. which clearly indicate that the stilbenes 8 are trans-isomers.

\section{Conclusions}

Unsymmetrical $t r a n s$-stilbenes are prepared via the sequential coupling reactions of bromobenzenesulfonate. The great chemoselectivity of the neopenyloxysulfonyl group, which is an excellent leaving group in the presence of a nickel catalyst while not reactive toward phosphonate carbanions or palladium catalyst at all. allows the stepwise construction of highly conjugated unsymmetrical stilbenes in good overall yields. Only trans-stilbenes are produced as a result of the stereoselectivity of the HWE reaction. To our knowledge, this is the first general preparation of unsymmetrical stilbenes possessing oligophenyl moieties, which would be useful as novel luminescent materials. The procedure described in this paper appears to be a promising and conceptually straightforward route for the parallel synthesis of various unfunctionalized hydrocarbons with phenyl and vinyl units. The synthesis and characterization of other highly conjugated compounds using this method are under investigation. and will be reported in due course

\section{Experimental Section}

General. Solvents were distilled from appropriate drying agents prior to use: THF and DME from sodium-benzophenone ketyl: toluene from $\mathrm{CaH}_{2}$. Commercially available reagents were used without further purification unless otherwise stated. ${ }^{1} \mathrm{H}$ NMR $(500 \mathrm{MHz})$ and ${ }^{13} \mathrm{C}$ NMR (125 $\mathrm{MHz}$ ) were registered in $\mathrm{CDCl}_{3}$ as solvent and tetramethylsilane (TMS) as internal standard. Chemical shifts are reported in $\delta$ units (ppm) by assigning TMS resonance in the ${ }^{1} \mathrm{H}$ and ${ }^{13} \mathrm{C}$ spectrum as $0.00 \mathrm{ppm}$. All coupling constants, $J$. are reported in hertz $(\mathrm{Hz})$. Columu chromatography was performed on silica gel 60. 70-230 mesh. Analytical thinlayer chromatography (TLC) was performed using Merck Kieselgel $60 \mathrm{~F}_{3 \mathrm{~s}}$ precoated plates $(0.25 \mathrm{~mm})$ with a fluorescent indicator and visualized with UV light ( 254 and 365 nun) or by iodine vapor staining. GC analy sis was performed on a bonded $5 \%$ phenylpolysiloxane BPX 5 capillary column (SGE. $30 \mathrm{~m} .0 .32 \mathrm{~mm}$ i.d.). Electron impact (EI. 70 $\mathrm{eV})$ was used as the ionization method for the mass spectrometry.

General Procedure for the Preparation of Stilbenesulfonates 6. To the mixture of benzyl phosphonates 5 (3.08 mmol) and sodium hydride (124 mg of $60 \%$ oil dispersion. $3.10 \mathrm{mmol})$ in DME (15 $\mathrm{mL})$ was added formylbiphenylsulfonates $4(2.80 \mathrm{mmol})$ in DME $(15 \mathrm{~mL})$ dropwise at room temperature under Ar atmosphere. The brown mixture was heated at reflux for 10-16 $\mathrm{h}$ until TLC analysis showed the complete disappearance of $\mathbf{4}$. The reaction mixture was cooled to room temperature and diluted with $\mathrm{CH}_{2} \mathrm{Cl}_{2}$. The resulting mixture was washed with $1 \%$ aqueous $\mathrm{HCl}$. water. and brine: dried over $\mathrm{MgSO}_{4}$ : and concentrated in vacuo. The crude product 6 was purified by recrystallization from $i$-PrOH or $i-\mathrm{PrOH}: n$-hexane $(90: 10)$.

(E)-2,2-Dimethyl-3-phenyl-1-propyl f'-styryl-1,1'-biphenyl-4-sulfonate (6a) was prepared by the reaction of $\mathbf{5 a}$ $(0.660$ g. $3.08 \mathrm{mmol}), \mathrm{NaH}(124 \mathrm{mg}$ of $60 \%$ oil dispersion, $3.10 \mathrm{mmol})$. and $+\mathrm{a}(1.14 \mathrm{~g} .2 .80 \mathrm{mmol})$. The crude product was purified by recrystallization from $i-\mathrm{PrOH}$ to afford $6 \mathrm{a}$ $\left(1.05\right.$ g. $78 \%$ ) as a pale yellowish solid: $\mathrm{mp} 134-135^{\circ} \mathrm{C}$ (uncorrected); TLC $R_{f} 0.16\left(\mathrm{CH}_{2} \mathrm{Cl}_{2}: n\right.$-hexane $\left.=1: 1\right) ;{ }^{1} \mathrm{H}$ NMR $\left(500 \mathrm{MHz}, \mathrm{CDCl}_{3}\right) \delta 0.90$ (s. $\left.6 \mathrm{H}\right), 2.57(\mathrm{~s}, 2 \mathrm{H}), 3.72$ $(\mathrm{s}, 2 \mathrm{H}) .7 .02$ (dd. $J=7.80,1.91 \mathrm{~Hz} .2 \mathrm{H}) .7 .18-7.2 \mathrm{l}(\mathrm{m}, 3 \mathrm{H})$, $7.19(\mathrm{~s}, 2 \mathrm{H}) .7 .29$ (t. $J=7.68 \mathrm{~Hz}, \mathrm{lH}) .7 .39$ (t. $J=7.63 \mathrm{~Hz}$, $2 \mathrm{H}$ ). 7.55 (d. $J=8.44$ Hz. $2 \mathrm{H}$ ). 7.64 (s. $4 \mathrm{H}), 7.79$ (d. $J=8.51$ Hz. $2 \mathrm{H}$ ). 7.99 (d. $J=8.5 \mathrm{l} \mathrm{Hz} .2 \mathrm{H}) ;{ }^{13} \mathrm{C}$ NMR $(125 \mathrm{MHz}$, $\left.\mathrm{CDCl}_{3}\right) \delta 24.1(\times 2), 35.3 .44 .3,77.2,126.3,126.7(\times 2)$, $127.2(\times 2) .127 .5(\times 2), 127.6(\times 2), 127.7 .128 .0(\times 2) .128 .0$, $128.5(\times 2) .128 .8(\times 2), 129.8 .130 .4(\times 2), 134.4 .137 .0$, 137.4, 137.9, 138.0, 146.1: HRMS (EI, $70 \mathrm{eV}$ ) calcd for $\mathrm{C}_{31} \mathrm{H}_{31} \mathrm{O}_{3} \mathrm{~S}\left(\mathrm{M}^{+}\right) 482.1916$. found 482.1838 .

(E)-2,2-Dimethyl-3-phenyl-1-propyl +'-[2-(4-tolyl)vinyl]1,1 '-biphenyl-4-sulfonate (6b) was prepared by the reaction of $\mathbf{5 b}(0.703$ g. $3.08 \mathrm{mmol})$. $\mathrm{NaH}(124 \mathrm{mg}$ of $60 \%$ oil dispersion, $3.10 \mathrm{mmol}$ ), and ta $(1.14 \mathrm{~g} .2 .80 \mathrm{mmol})$. The crude product was purified by recrystallization from $i-\mathrm{PrOH}$ to afford $6 \mathbf{b}(1.06 \mathrm{~g} .76 \%)$ as a bright yellow solid: $\mathrm{mp} 198$ $199^{\circ} \mathrm{C}$ (uncorrected): TLC $R_{f} 0.16\left(\mathrm{CH}_{2} \mathrm{Cl}_{2}: n\right.$-hexane $\left.=1: 1\right)$ : ${ }^{1} \mathrm{H}$ NMR $\left(500 \mathrm{MHz}, \mathrm{CDCl}_{3}\right) \delta 0.90$ (s. $6 \mathrm{H}$ ). 2.38 (s. $\left.3 \mathrm{H}\right)$, 2.57 (s. $2 \mathrm{H}), 3.72$ (s. $2 \mathrm{H}), 7.02$ (dd. $J=7.80 .1 .91 \mathrm{~Hz} .2 \mathrm{H}$ ), $7.1 \mathrm{l}(\mathrm{d}, J=16.31 \mathrm{~Hz}, \mathrm{lH}) .7 .167 .2 \mathrm{l}(\mathrm{m}, 6 \mathrm{H}) .7 .45$ (d. $J=$ $8.06 \mathrm{~Hz}, 2 \mathrm{H}), 7.63(\mathrm{~s}, 4 \mathrm{H}), 7.79(\mathrm{~d} . J=8.52 \mathrm{~Hz}, 2 \mathrm{H}), 7.99$ (d. $J=8.52 \mathrm{~Hz}, 2 \mathrm{H}){ }^{13} \mathrm{C}$ NMR $\left(125 \mathrm{MHz} . \mathrm{CDCl}_{3}\right) \delta 21.3$, $24.1(\times 2), 35.3,44.3 .77 .2,126.3 .126 .6(\times 2) .126 .7,127.1$ $(\times 2) .127 .5(\times 2) .127 .6(\times 2), 128.0(\times 2), 128.5(\times 2), 129.5$ $(\times 2) .129 .8,130.4(\times 2), 134.3,134.4,137.4 .137 .7,138.0$, 138.2, 146.2: HRMS (EI, $70 \mathrm{eV}$ ) calcd for $\mathrm{C}_{32} \mathrm{H}_{32} \mathrm{O}_{3} \mathrm{~S}\left(\mathrm{M}^{-}\right)$ 496.2072 , found 496.2008 .

(E)-2,2-Dimethyl-3-phenyl-1-propyl 4'-[2-(4-tert-butylphenyl)vinyl]-1,1'-biphenyl-4-sulfonate ( $6 \mathrm{c}$ ) was prepared by the reaction of $5 \mathrm{c}(0.833 \mathrm{~g} .3 .08 \mathrm{mmol})$. NaH (124 mg of $60 \%$ oil dispersion, 3.10 mmol). and $4 \mathrm{a}(1.14 \mathrm{~g} .2 .80 \mathrm{mmol})$. The crude product was purified by recrystallization from $i$-PrOH to afford $6 \mathrm{c}(1.10 \mathrm{~g} .73 \%)$ as a fluffy white solid: $\mathrm{mp}$ $158-159^{\circ} \mathrm{C}$ (uncorrected): $\mathrm{TLC} R_{f} 0.19\left(\mathrm{CH}_{2} \mathrm{Cl}_{2}\right.$ :n-hexane $=$ $1: 1) ;{ }^{1} \mathrm{H} \mathrm{NMR}\left(500 \mathrm{MHz} . \mathrm{CDCl}_{3}\right) \delta 0.90(\mathrm{~s}, 6 \mathrm{H}) .1 .35(\mathrm{~s}$, $9 \mathrm{H}) .2 .57(\mathrm{~s}, 2 \mathrm{H}) .3 .72(\mathrm{~s}, 2 \mathrm{H}) .7 .02(\mathrm{dd}, J=7.80,1.93 \mathrm{~Hz}$, 2H). $7.12(\mathrm{~d} . J=16.30 \mathrm{~Hz}, 1 \mathrm{H}) .7 .167 .21(\mathrm{~m} .4 \mathrm{H}), 7.41$ (d. $J$ $=8.41 \mathrm{~Hz}, 2 \mathrm{H}) .7 .49(\mathrm{~d} J=8.41 \mathrm{~Hz}, 2 \mathrm{H}) .7 .63(\mathrm{~s} .4 \mathrm{H}) .7 .79$ (d. $J=8.51 \mathrm{~Hz}, 2 \mathrm{H}) .7 .99(\mathrm{~d} J=8.51 \mathrm{~Hz}, 2 \mathrm{H}):{ }^{13} \mathrm{C}$ NMR $(125 \mathrm{MHz} . \mathrm{CDCl}$ ) $\delta 24.1(\times 2), 31.3(\times 3), 34.7,35.3,44.3$, $77.2,125.7(\times 2) .126 .3 .126 .4(\times 2) .126 .9,127.1(\times 2), 127.5$ $(\times 2) .127 .6(\times 2), 127.9(\times 2), 128.5(\times 2), 129.7,130.4(\times 2)$. 134.3, 134.4. 137.4, 137.7, 138.2, 146.2. 151.2: HRMS (EI. $70 \mathrm{eV}$ ) calcd for $\mathrm{C}_{33} \mathrm{H}_{38} \mathrm{O}_{3} \mathrm{~S}\left(\mathrm{M}^{+}\right) 538.2542$, found 538.2527 .

(E)-2,2-Dimethyl-3-phenyl-1-propyl 4'-[2-(2-methylbiphenyl-3-yl)vinyl]-1,1'-biphenyl-4-sulfonate (6d) was prepared by the reaction of $5 d(0.536 \mathrm{~g} .1 .76 \mathrm{mmol}) . \mathrm{NaH}$ 
( $72.0 \mathrm{mIg}$ of $60 \%$ oil dispersion. $1.80 \mathrm{mmol}$ ), and ta $(0.654$ g. 1.60 mmol). The crude product was purified by recrystallization to from $i-\mathrm{PrOH}$ afford $6 \mathrm{~d}(0.70 \mathrm{~g} .76 \%)$ as a yellow solid: mp 143-145 ${ }^{\circ} \mathrm{C}$ (uncorrected): ${ }^{1} \mathrm{H}$ NMR $(500 \mathrm{MHz}$. $\left.\mathrm{CDCl}_{3}\right) \delta 0.90$ (s. $6 \mathrm{H}$ ). 2.33 (s, 3H), $2.57(\mathrm{~s}, 2 \mathrm{H}), 3.72$ (s. $2 \mathrm{H}), 7.02(\mathrm{dd}, J=7.74,1.95 \mathrm{~Hz} .2 \mathrm{H}), 7.07(\mathrm{~d}, J=16.08 \mathrm{~Hz}$. lH), 7.16-7.21 (m, 4H), $7.28(\mathrm{t}, J=7.64 \mathrm{~Hz} . \mathrm{lH}) .7 .32(\mathrm{~d} . J$ $=7.53 \mathrm{~Hz}, 2 \mathrm{H}), 7.36(\mathrm{t} . J=7.44 \mathrm{~Hz}, 1 \mathrm{H}), 7.43(\mathrm{t} . J=7.37$ $\mathrm{Hz}, 2 \mathrm{H}) .7 .52(\mathrm{~d} . J=16.08 \mathrm{~Hz} .1 \mathrm{H}), 7.62(\mathrm{~d}, J=7.28 \mathrm{~Hz}$. $1 \mathrm{H}), 7.65(\mathrm{~d}, J=8.76 \mathrm{~Hz} .2 \mathrm{H}), 7.67(\mathrm{~d}, J=8.76 \mathrm{~Hz}, 2 \mathrm{H})$. $7.79(\mathrm{~d}, J=8.42 \mathrm{~Hz} .2 \mathrm{H}), 7.99(\mathrm{~d} . J=8.42 \mathrm{~Hz}, 2 \mathrm{H}){ }^{13} \mathrm{C}$ NMR $\left(125 \mathrm{MHz}, \mathrm{CDCl}_{3}\right) \delta 17.2,24.1(\times 2), 35.3,44.3,77.2$. 125.0. 125.8, 126.3. 126.9, $127.3(\times 2), 127.5(\times 2) .127 .7$ $(\times 2), 128.0(\times 2), 128.1(\times 2), 128.5,128.5(\times 2), 129.4(\times 2)$. 129.7. 129.8. 130.4 ( $\times 2), 133.4$. 134.5. 137.1, 137.4, 138.0. 138.3. 142.2. 143.0. 146.1; HRMS (EI. $70 \mathrm{eV}$ ) calcd for $\mathrm{C}_{38} \mathrm{H}_{36} \mathrm{O}_{3} \mathrm{~S}\left(\mathrm{M}^{+}\right)$572.2385. found 572.2335 .

(E)-2,2-Dimethyl-3-phenyl-1-propyl 4'-methoxy-3'styryl-1,1'-biphenyl-t-sulfonate (6e) was prepared by the reaction of $5 \mathrm{a}(0.259 \mathrm{~g} .1 .21 \mathrm{mmol})$. $\mathrm{NaH}(50.0 \mathrm{mg}$ of $60 \%$ oil dispersion, $1.25 \mathrm{mmol})$. and $+\mathrm{b}(0.482 \mathrm{~g}, 1.10 \mathrm{mmol})$. The crude product was purified by recrystallization from $i-\mathrm{PrOH} n$-hexane $(9: 1)$ to afford $6 \mathrm{e}(0.31 \mathrm{~g} .55 \%)$ as a pale yellowish solid: mp $124-126^{\circ} \mathrm{C}$ (uncorrected): TLC $R_{f} 0.25$ $\left(\mathrm{CH}_{2} \mathrm{Cl}\right.$ : $n$-hexane $\left.=1: 1\right):{ }^{1} \mathrm{H}$ NMR $\left(500 \mathrm{MHz}, \mathrm{CDCl}_{3}\right) \delta$ 0.90 (s, 6H), 2.57 (s. $2 \mathrm{H}$ ). 3.72 (s, 2H), 3.96 (s. $3 \mathrm{H}$ ). 7.02 (d. $J=8.59 \mathrm{~Hz}, \mathrm{LH}) .7 .03(\mathrm{dd}, J=7.86 .1 .82 \mathrm{~Hz}, 2 \mathrm{H}), 7.16-7.20$ $(\mathrm{m}, 3 \mathrm{H}), 7.20($ d. $J=16.45 \mathrm{~Hz} .1 \mathrm{H}), 7.27(\mathrm{t}, J=7.34 \mathrm{H}, 1 \mathrm{H})$. $7.37(\mathrm{t}, J=7.66 \mathrm{~Hz}, 2 \mathrm{H}), 7.5 \mathrm{~L}(\mathrm{~d} . J=16.45 \mathrm{~Hz} .1 \mathrm{H}) .7 .5 \mathrm{l}$ (dd. $J=8.59,2.35 \mathrm{~Hz} .1 \mathrm{H}), 7.56($ d. $J=7.34 \mathrm{~Hz} .2 \mathrm{H}), 7.78$ (d. $J=8.50 \mathrm{~Hz}, 2 \mathrm{H}), 7.84$ (d. $J=2.35 \mathrm{~Hz} . \mathrm{lH}) .7 .98(\mathrm{~d}, J=$ $8.50 \mathrm{~Hz} .2 \mathrm{H}):{ }^{13} \mathrm{C}$ NMR $\left(125 \mathrm{MHz}, \mathrm{CDCl}_{3}\right) \delta 24.1(\times 2)$. $35.3,44.3 .55 .8 .77 .2$. 111.5. 123.0, 125.4, 126.3. $126.7(\times 2)$. 127.2. $127.3(\times 2), 127.5,127.7,128.0(\times 2), 128.5(\times 2)$. $128.7(\times 2) .130 .1,130.5(\times 2), 131.5 .133 .9,137.4,137.6$. 146.4. 157.6: HRMS (EI. $70 \mathrm{eV}$ ) calcd for $\mathrm{C}_{32} \mathrm{H}_{32} \mathrm{O}_{4} \mathrm{~S}(\mathrm{M}+$ ) 512.2021 . found 512.2033 .

(E)-2,2-Dimethyl-3-phenyl-1-propyl 4'-methoxy-3'-[2(4-tolyl)vinyl]-1,1'-biphenyl-4-sulfonate (6f) was prepared by the reaction of $5 \mathrm{~b}(0.276 \mathrm{~g} .1 .21 \mathrm{mmol}) . \mathrm{NaH}(50.0 \mathrm{mg}$ of $60 \%$ oil dispersion. $1.25 \mathrm{mmol})$ and $4 \mathrm{~b}(0.428 \mathrm{~g} .1 .10$ $\mathrm{mmol}$ ). The crude product was purified by recrystallization to from $i$-PrOH: $n$-hexane $(9: 1)$ afford $6 f(0.250 \mathrm{~g} .43 \%)$ as a white solid: $\mathrm{mp}$ 96-97 ${ }^{\circ} \mathrm{C}$ (uncorrected); TLC $R_{f} 0.26$ $\left(\mathrm{CH}_{3} \mathrm{Cl}_{2}\right.$ n-hexane $\left.=1: 1\right):{ }^{1} \mathrm{H}$ NMR $\left(500 \mathrm{MHz}, \mathrm{CDCl}_{3}\right) \delta$ 0.90 (s. $6 \mathrm{H}$ ), 2.37 (s. $3 \mathrm{H}$ ). 2.57 (s. $2 \mathrm{H}$ ). 3.72 (s. $2 \mathrm{H}$ ). 3.95 (s. $3 \mathrm{H}), 7.00-7.03$ (m. $3 \mathrm{H}) .7 .16-7.20$ (m. $6 \mathrm{H}), 7.46$ (d. $J=$ $16.46 \mathrm{~Hz}, \mathrm{lH}) .7 .46$ (d. $J=8.07 \mathrm{~Hz}, 2 \mathrm{H}) .7 .50$ (dd $J=8.50$. $2.31 \mathrm{~Hz}, \mathrm{lH}) .7 .78(\mathrm{~d} . J=8.49 \mathrm{~Hz}, 2 \mathrm{H}) .7 .84(\mathrm{~d} . J=2.31 \mathrm{~Hz}$. 1H). $7.98($ d. $J=8.49 \mathrm{~Hz}, 2 \mathrm{H}){ }^{13} \mathrm{C} \mathrm{NMR}\left(125 \mathrm{MHz} . \mathrm{CDCl}_{3}\right)$ $\delta 21.3 .24 .1(\times 2) .35 .3,44.3,55.8,77.2 .111 .4,121.9 .125 .3$. 126.3. $126.6(\times 2), 127.3,127.3(\times 2), 127.4,128.0(\times 2)$. $128.5(\times 2) .129 .4(\times 2), 130.0 .130 .4(\times 2), 131.5,133.8$. 134.8. 137.4. 137.6. 146.4. 157.5: HRMS (EI. $70 \mathrm{eV}$ ) calcd for $\mathrm{C}_{33} \mathrm{H}_{34} \mathrm{O}_{4} \mathrm{~S}(\mathrm{M}+)$ 526.2178. found 526.2162 .

(E)-2,2-Dimethyl-3-phenyl-1-propyl 3'-[2-(4-tert-butylphenyl)vinyl]-4'-methoxy-1,1'-biphenyl-4-sulfonate (6g) was prepared by the reaction of $5 \mathrm{c}(0.327 \mathrm{~g} .1 .21 \mathrm{mmol})$, $\mathrm{NaH}$ (50.0 mg of $60 \%$ oil dispersion. $1.25 \mathrm{mmol})$. and $\mathbf{t b}$ $(0.482 \mathrm{~g} .1 .10 \mathrm{mmol})$. The crude product was purified by recrystallization to from $i-\mathrm{PrOH} n$-hexane $(9: 1)$ afford $\mathbf{6 g}$ $(0.260 \mathrm{~g}, 41 \%)$ as a fluffy white solid: $\mathrm{mp} 117118^{\circ} \mathrm{C}$ (uncorrected); TLC $\mathrm{R}_{f} 0.30\left(\mathrm{CH}_{2} \mathrm{Cl}_{2}: 77\right.$-hexane $\left.=1: 1\right) ;{ }^{1} \mathrm{H}$ NMR (500 MHz, CDCl $) \delta 0.90$ (s. 6H), $1.34(\mathrm{~s}, 9 \mathrm{H}), 2.57$ (s, 2H), 3.72 (s. 2H). 3.95 (s, 3H). 7.00-7.04 (m, 3H). 7.16$7.2 \mathrm{l}(\mathrm{m}, 4 \mathrm{H}), 7.40(\mathrm{~d} . J=8.40 \mathrm{~Hz}, 2 \mathrm{H}), 7.47(\mathrm{~d}, J=16.93$ Hz. $\mathrm{lH}$ ), $7.49-7.5 \mathrm{l}(\mathrm{m}, 3 \mathrm{H}) .7 .78(\mathrm{~d}, J=8.49 \mathrm{~Hz} .2 \mathrm{H}), 7.84$ $($ d. $J=2.28 \mathrm{~Hz} . \mathrm{lH}), 7.98$ (d. $J=8.49 \mathrm{~Hz}, 2 \mathrm{H}) ;{ }^{13} \mathrm{C}$ NMR $\left(125 \mathrm{MHz} . \mathrm{CDCl}_{3}\right) \delta 24.1(\times 2), 31.3(\times 3), 34.7,35.3,44.3$, 55.8. 77.2. 111.5. 122.2. 125.3. $125.6(\times 2) .126 .3,126.4$ $(\times 2) .127 .3,127.3(\times 2), 127.4,128.0(\times 2), 128.5(\times 2), 129.8$. $130.5(\times 2) .131 .5,133.8,134.9 .137 .4 .146 .5,150.9,157.5$ : HRMS (EI. $70 \mathrm{eV}$ ) calcd for $\mathrm{C}_{36} \mathrm{H}_{40} \mathrm{O}_{4} \mathrm{~S}\left(\mathrm{M}^{-}\right) 568.2647$, found 568.2636 .

General Procedure for the Preparation of Unsymmetrical Stilbenes 8. To a stirred solution of $6(0.100 \mathrm{mmol})$ and dppf $\mathrm{NiCl}_{2}(3.42 \mathrm{mg} .0 .00500 \mathrm{mmol})$ in dry THF (20 $\mathrm{mL}$ ) were added the aryl Grignard reagents $7(0.30 \mathrm{mmol})$ via a syringe at room temperature. This resulted in a color change from dark green to dark brown. The reaction mixture was heated under reflux for $6 \mathrm{~h}$ and then cooled to room temperature, and an additional $0.20 \mathrm{mmol}$ of 7 was added. The reaction mixture was stirred under reflux for a further 6 $h$. The reaction mixture was poured into a separatory funnel containing $\mathrm{CH}_{2} \mathrm{Cl}_{2}$ and $1 \%$ aqueous $\mathrm{HCl}$. The combined organic layer was washed with water and brine, dried over $\mathrm{MgSO}_{4}$. and concentrated in vacto. To the concentrated mixture was added $\mathrm{MeOH}$ to precipitate the product 8 . The suspension was filtered through a small pad of silica gel in a sintered glass filter. The solid remaining on the silica gel was washed with $\mathrm{MeOH}$. collected by elution with $\mathrm{CH}_{2} \mathrm{Cl}_{2}$. and concentrated under reduced pressure. The resulting crude product was purified by column chromatograply.

(E)-t-Styryl-[1,1'; $+^{\prime}, 1^{\prime \prime} \mid$ terphenyl (8a) was prepared by the reaction of $6 \mathrm{a}(48.3 \mathrm{mg} .0 .100 \mathrm{mmol})$ with $7 \mathrm{a}(1.0 \mathrm{M}$ in THF. $0.30 \mathrm{~mL} .0 .30 \mathrm{mmol}+0.20 \mathrm{~mL} .0 .20 \mathrm{mmol}$ ) in the presence of $\mathrm{dppfNiCl}$. The crude compound was purified by column chromatography $\left(\mathrm{CH}_{2} \mathrm{Cl}_{2}\right)$ to give $8 \mathrm{a}(13.6 \mathrm{mg}$. $41 \%$ ) as a pale ivory solid: $\mathrm{mp} 315-317^{\circ} \mathrm{C}$ (uncorrected): TLC $R_{f} 0.57\left(\mathrm{CH}_{2} \mathrm{Cl}\right.$ : -hexane $\left.=1: 1\right) ;{ }^{1} \mathrm{H}$ NMR $(500 \mathrm{MHz}$, $\left.\mathrm{CDCl}_{3}\right) \delta 7.17$ (s. $2 \mathrm{H}$ ). 7.28 (t. $J=7.39 \mathrm{~Hz}, \mathrm{lH}$ ), $7.35-7.39$ $(\mathrm{m}, 3 \mathrm{H}) .7 .47$ (t. $J=7.57 \mathrm{~Hz} .2 \mathrm{H}), 7.55($ d. $J=7.47 \mathrm{~Hz} .2 \mathrm{H}$ ), $7.61(\mathrm{~d} . J=8.21 \mathrm{~Hz}, 2 \mathrm{H}) .7 .65(\mathrm{~d} . J=7.57 \mathrm{~Hz}, 2 \mathrm{H}) .7 .66(\mathrm{~d}$. $J=8.21 \mathrm{~Hz} .2 \mathrm{H}$ ). 7.69 (d. $J=8.37 \mathrm{~Hz} .2 \mathrm{H}$ ). 7.72 (d. $J=8.37$ Hz. 2H): HRMS (EI. $70 \mathrm{eV}$ ) calcd for $\mathrm{C}_{36} \mathrm{H}_{2 \mathrm{il}}\left(\mathrm{M}^{+}\right) 332.1565$. found 332.1520 .

(E)-4"-Methyl-4-styryl-[1,1';4',1"]terphenyl $(8 \mathrm{~b})$ was prepared by the reaction of $6 \mathrm{a}(48.3 \mathrm{mg} .0 .100 \mathrm{mmol})$ with $7 \mathrm{~b}\left(1.0 \mathrm{M}\right.$ in $\mathrm{Et}_{2} \mathrm{O}, 0.30 \mathrm{~mL}, 0.30 \mathrm{mmol}+0.20 \mathrm{~mL} .0 .20$ munol) in the presence of dppfNiCl . The crude compound was purified by colunn chromatography $\left(\mathrm{CH}_{2} \mathrm{Cl}_{2}\right)$ to give $8 \mathbf{b}(19.4 \mathrm{mg}, 56 \%)$ as an ivory solid: $\mathrm{mp} 326.328{ }^{\circ} \mathrm{C}$ (uncorrected); TLC $R_{f} 0.57\left(\mathrm{CH}_{2} \mathrm{Cl}_{2}: n\right.$-hexane $\left.=1: 1\right) ;{ }^{1} \mathrm{H}$ $\operatorname{NMR}\left(500 \mathrm{MHz} . \mathrm{CDCl}_{3}\right) \delta 2.41(\mathrm{~s}, 3 \mathrm{H}) .7 .17(\mathrm{~s} .2 \mathrm{H}), 7.26-$ 
$7.29(\mathrm{~m}, 3 \mathrm{H}) .7 .38(\mathrm{t}, J=7.65 \mathrm{~Hz}, 2 \mathrm{H}) .7 .55(\mathrm{~d} . J=8.33 \mathrm{~Hz}$. $2 \mathrm{H}) .7 .55$ (d. $J=8.06 \mathrm{~Hz} .2 \mathrm{H}), 7.6 \mathrm{l}($ d. $J=8.28 \mathrm{~Hz}, 2 \mathrm{H})$. $7.66($ d. $J=8.28 \mathrm{~Hz}, 2 \mathrm{H}) .7 .67$ (d. $J=8.34 \mathrm{~Hz}, 2 \mathrm{H}) .7 .70$ (d. $J=8.34 \mathrm{~Hz}, 2 \mathrm{H}$ ); HRMS $\left(\mathrm{EI} .70 \mathrm{eV}\right.$ ) calcd for $\mathrm{C}_{27} \mathrm{H}_{22}\left(\mathrm{M}^{+}\right)$ 346.1721 . found 346.1755

(E)-4"-tert-Butyl-4-styryl-[1,1';4',1"']terphenyl (8c) was prepared by the reaction of $6 \mathbf{a}(48.3 \mathrm{mg}, 0.100 \mathrm{~nm}$ ( $)$ with $7 \mathrm{c}(2.0 \mathrm{M}$ in Et:O $0.15 \mathrm{~mL} .0 .30 \mathrm{mmol}+0.10 \mathrm{~mL} .0 .20$ mmol) in the presence of dppfNiCl. The crude compound was purified by colunun chromatography $\left(\mathrm{CH}_{2} \mathrm{Cl}_{2}\right)$ to give $8 \mathrm{c}$ (19.8 mg. $51 \%$ ) as a pale yellow solid: $\mathrm{mp} 279-283{ }^{\circ} \mathrm{C}$ (uncorrected): TLC $R_{f} 0.61\left(\mathrm{CH}_{2} \mathrm{Cl}:\right.$-h-hexane $\left.=1: 1\right):{ }^{1} \mathrm{H}$ NMR (500 MHz. $\left.\mathrm{CDCl}_{3}\right) \delta$ I.38 (s. 9H). 7.17 (s. $2 \mathrm{H}$ ). 7.28 (t. $J=7.45$ Hz. IH). 7.38 (t. $J=7.63 \mathrm{~Hz} .2 \mathrm{H}$ ). 7.49 (d. $J=$ $8.38 \mathrm{~Hz}, 2 \mathrm{H}), 7.55(\mathrm{~d} . J=7.30 \mathrm{~Hz}, 2 \mathrm{H}), 7.60(\mathrm{~d}, J=8.38 \mathrm{~Hz}$. $2 \mathrm{H}), 7.6 \mathrm{l}(\mathrm{d}, J=8.49 \mathrm{~Hz} .2 \mathrm{H}), 7.66(\mathrm{~d}, J=8.49 \mathrm{~Hz}, 2 \mathrm{H})$. $7.68(\mathrm{~d} . J=8.48 \mathrm{~Hz} .2 \mathrm{H}), 7.70(\mathrm{~d} . J=8.48 \mathrm{~Hz}, 2 \mathrm{H})$ : HRMS (EI. $70 \mathrm{eV}$ ) calcd for $\mathrm{C}_{30} \mathrm{H}_{28}\left(\mathrm{M}^{+}\right) 388.2191$. found $388.2 \mathrm{l} 46$.

(E)-t-[2-(+-Tolyl)viny]]-[1,1'; ', 1' ']terphenyl (8d) was prepared by the reaction of $6 \mathbf{b}(49.7 \mathrm{mg} .0 .100 \mathrm{~mm}$ ol) with 7 a $(1.0 \mathrm{M}$ in THF, $0.30 \mathrm{~mL} .0 .30 \mathrm{mmol}+0.20 \mathrm{~mL}, 0.20$ mmol) in the presence of dppfNiCl. The crude compound was purified by column chromatography $\left(\mathrm{CH}_{2} \mathrm{Cl}_{2}\right)$ to give $8 d(21.8 \mathrm{mg}, 63 \%)$ as a pale yellow solid: $\mathrm{mp} 313-316{ }^{\circ} \mathrm{C}$ (uncorrected): TLC $R_{f} 0.58\left(\mathrm{CH}_{2} \mathrm{Cl}\right.$ : $:$-hexane $\left.=1: 1\right):{ }^{1} \mathrm{H}$ NMR $\left(500 \mathrm{MHz}, \mathrm{CDCl}_{3}\right) \delta 2.37$ (s. $\left.3 \mathrm{H}\right), 7.11$ (d. $J=16.38$ $\mathrm{Hz}, \mathrm{lH}) .7 .15(\mathrm{~d}, J=16.38 \mathrm{~Hz} . \mathrm{H}), 7.19(\mathrm{~d}, J=7.81 \mathrm{~Hz}$. $2 \mathrm{H}$ ). 7.37 (t. $J=7.38 \mathrm{lH}$ ). 7.44 (d. $J=7.81$ Hz. $2 \mathrm{H}$ ). 7.47 (t. $J=7.45 \mathrm{~Hz}, 2 \mathrm{H}) .7 .60(\mathrm{~d} . J=8.2 \mathrm{l} \mathrm{Hz} .2 \mathrm{H}) .7 .65$ (d. $J=7.91$ $\mathrm{Hz}, 4 \mathrm{H}), 7.68(\mathrm{~d}, J=8.27 \mathrm{~Hz} .2 \mathrm{H}) .7 .7 \mathrm{l}(\mathrm{d}, J=8.27 \mathrm{~Hz}$. 2H): HRMS (EI, $70 \mathrm{eV}$ ) calcd for $\mathrm{C}_{27} \mathrm{H}_{22}\left(\mathrm{M}^{+}\right) 346.1712$. found 346.1724 .

(E)-4"-Methyl-t-[2-(t-tolyl)viny]]-[1,1'; $\left.t^{\prime}, 1^{\prime \prime}\right]$ terphenyl (8e) was prepared by the reaction of $6 \mathbf{b}(49.7 \mathrm{mg}, 0.100$ mmol) with $7 \mathrm{~b}\left(1.0 \mathrm{M}\right.$ in $\mathrm{Et}_{2} \mathrm{O} .0 .30 \mathrm{~mL}, 0.30 \mathrm{mmol}+0.20$ $\mathrm{mL} .0 .20 \mathrm{mmol}$ ) in the presence of dppf $\mathrm{NiCl}_{2}$. The crude comipound was purified by column chronatography $\left(\mathrm{CH}_{2} \mathrm{Cl}_{2}\right)$ to give $8 \mathrm{e}(24.9 \mathrm{mg} .69 \%)$ as a pale irory solid: $\mathrm{mp} 326-330$ ${ }^{\circ} \mathrm{C}$ (uncorrected): TLC $R_{f} 0.59\left(\mathrm{CH}_{3} \mathrm{Cl}_{2}\right.$-h -hexane $\left.=1: 1\right):{ }^{1} \mathrm{H}$ NMR $\left(500 \mathrm{MHz}, \mathrm{CDCl}_{3}\right) \delta 2.36$ (s, 3H), 2.37 (s. $\left.3 \mathrm{H}\right) .7 .11$ (d. $J=16.41 \mathrm{~Hz} .1 \mathrm{H}$ ). 7.15 (d. $J=16.41 \mathrm{~Hz}, 1 \mathrm{H}$ ). 7.19 (d. $J$ $=7.94 \mathrm{~Hz}, 2 \mathrm{H}) .7 .28(\mathrm{~d} J=8.11 \mathrm{~Hz}, 2 \mathrm{H}) .7 .44(\mathrm{~d} . J=7.94$ Hz, $2 \mathrm{H}), 7.55(\mathrm{~d} J=8.11 \mathrm{~Hz}, 2 \mathrm{H}), 7.59(\mathrm{~d}, J=8.17 \mathrm{~Hz}, 2 \mathrm{H})$. 7.65 (d. $J=8.17 \mathrm{~Hz}, 2 \mathrm{H}) .7 .67($ d. $J=8.59 \mathrm{~Hz}, 2 \mathrm{H}) .7 .70(\mathrm{~d}$. $J=8.59 \mathrm{~Hz}, 2 \mathrm{H})$ : HRMS (EI. $70 \mathrm{eV}$ ) calcd for $\mathrm{C}_{3 \mathrm{~S}} \mathrm{H}_{\mathrm{Z}^{+}}\left(\mathrm{M}^{+}\right)$ 360.1878 . found 360.1840 .

(E)-4"'-tert-Buty]-4-[2-(4-tolyl) vinyl]-[1,1';4',1"]terphenyl (8f) was prepared by the reaction of $6 \mathbf{b}(49.7 \mathrm{mg} .0 .100$ mmol) with $7 \mathrm{c}\left(2.0 \mathrm{M}\right.$ in $\mathrm{Et}_{2} \mathrm{O}, 0.15 \mathrm{~mL}, 0.30 \mathrm{mmol}+0.10$ $\mathrm{mL} .0 .20 \mathrm{mmol}$ ) in the presence of dppf $\mathrm{NiCl}_{2}$. The crude compound was purified by column chromatography $\left(\mathrm{CH}_{2} \mathrm{Cl}_{2}\right)$ to give $8 f(27.0 \mathrm{mg}, 67 \%)$ as a pale yellow solid: $\mathrm{mp} 299$. $301^{\circ} \mathrm{C}$ (uncorrected): TLC $R_{f} 0.61\left(\mathrm{CH}_{2} \mathrm{Cl}_{2} ; n\right.$-hexane $\left.=1: 1\right)$ : ${ }^{1} \mathrm{H}$ NMR (500 MHz, $\mathrm{CDCl}_{3}$ ) $\delta 1.38$ (s, 9H). 2.37 (s, 3H). $7.11(\mathrm{~d}, J=16.48 \mathrm{~Hz}, 1 \mathrm{H}) .7 .15(\mathrm{~d} . J=16.48 \mathrm{~Hz} . \mathrm{LH}) .7 .19$ (d. $J=7.79 \mathrm{~Hz}, 2 \mathrm{H}) .7 .44$ (d. $J=7.79 \mathrm{~Hz}, 2 \mathrm{H}) .7 .49$ (d. $J=$ $8.17 \mathrm{~Hz}, 2 \mathrm{H}$ ). 7.59 (d. $J=7.70 \mathrm{~Hz}, 4 \mathrm{H}) .7 .65$ (d. $J=8.11 \mathrm{~Hz}$.
2H). $7.67($ d. $J=8.58 \mathrm{~Hz}, 2 \mathrm{H}$ ). $7.70(\mathrm{~d}, J=8.58 \mathrm{~Hz}, 2 \mathrm{H})$; HRMS (EI, $70 \mathrm{eV}$ ) calcd for $\mathrm{C}_{31} \mathrm{H}_{3 !}\left(\mathrm{M}^{+}\right) 402.2348$. found 402.2319 .

(E)-4-[2-(4-tert-Butylphenyl)vinyl]-[1,1';4',1 "]terphenyl (8g) was prepared by the reaction of $6 c(53.9 \mathrm{mg} .0 .100$ mumol) with 7 a ( $1.0 \mathrm{M}$ in THF, $0.30 \mathrm{~mL}, 0.30 \mathrm{mmol}+0.20$ $\mathrm{mL}, 0.20 \mathrm{mmol}$ ) in the presence of $\mathrm{dppfNiCl}_{2}$. The crude compound was purified by column chromatography $\left(\mathrm{CH}_{2} \mathrm{Cl}_{2}\right)$ to give $8 \mathrm{~g}$ (23.7 $\mathrm{mg} .61 \%$ ) as an ivory solid: $\mathrm{mp} 293.294^{\circ} \mathrm{C}$ (uncorrected); TLC $R_{f} 0.60\left(\mathrm{CH}_{2} \mathrm{Cl}_{2}: n\right.$-hexane $\left.=1: 1\right) ;{ }^{1} \mathrm{H}$ NMR $\left(500 \mathrm{MHz}\right.$. $\left.\mathrm{CDCl}_{3}\right) \delta 1.35$ (s. $\left.9 \mathrm{H}\right), 7.12$ (d. $J=16.37$ Hz. $1 \mathrm{H}), 7.17(\mathrm{~d}, J=16.37 \mathrm{~Hz} . \mathrm{lH}), 7.37$ (t. $J=7.40 \mathrm{~Hz}$, 1H). 7.40 (d. $J=8.27 \mathrm{~Hz}, 2 \mathrm{H}), 7.47($ t. $J=7.82 \mathrm{~Hz}, 2 \mathrm{H}$ ), $7.49(\mathrm{~d}, J=8.27 \mathrm{~Hz}, 2 \mathrm{H}), 7.60(\mathrm{~d}, J=8.28 \mathrm{~Hz} .2 \mathrm{H}), 7.65(\mathrm{~d}$. $J=8.25 \mathrm{~Hz} .4 \mathrm{H}), 7.68$ (d. $J=8.49 \mathrm{~Hz}, 2 \mathrm{H}) .7 .7 \mathrm{l}(\mathrm{d} . J=8.49$ Hz. 2H): HRMS (EI, $70 \mathrm{eV}$ ) calcd for $\mathrm{C}_{30} \mathrm{H}_{2},\left(\mathrm{M}^{-}\right)$ 388.2191 , found 388.2103 .

(E)-4-[2-(4-tert-Butylphenyl)vinyl]-4"--methyl-[1,1';4',1"]terphenyl (8h) was prepared by the reaction of $6 \mathrm{c}(53.9 \mathrm{mg}$, $0.100 \mathrm{mmol})$ with $7 \mathrm{~b}\left(1.0 \mathrm{M}\right.$ in $\mathrm{Et}_{2} \mathrm{O} .0 .30 \mathrm{~mL}, 0.30 \mathrm{mmol}+$ $0.20 \mathrm{~mL}, 0.20 \mathrm{mmol}$ ) in the presence of dppfNiCl . The crude compound was purified by column chromatography $\left(\mathrm{CH}_{2} \mathrm{Cl}_{2}\right)$ to give $8 \mathbf{h}(29.8 \mathrm{mg} .74 \%)$ as a pale yellow solid: $\mathrm{mp} 310-315{ }^{\circ} \mathrm{C}$ (uncorrected); TLC $R_{f} 0.64\left(\mathrm{CH}_{2} \mathrm{Cl}_{2}\right.$ : $n$-hexane $=1: 1):{ }^{1} \mathrm{H}$ NMR $\left(500 \mathrm{MHz} . \mathrm{CDCl}_{3}\right) \delta 1.35(\mathrm{~s} .9 \mathrm{H})$, $2.4 \mathrm{l}(\mathrm{s} .3 \mathrm{H}) .7 .12(\mathrm{~d} . J=16.3 \mathrm{l} \mathrm{Hz}, 1 \mathrm{H}) .7 .16(\mathrm{~d}, J=16.3 \mathrm{l}$ Hz. 1 H). 7.27 (d. $J=8.08$ Hz. $2 \mathrm{H}) .7 .40($ d. $J=8.37 \mathrm{~Hz}, 2 \mathrm{H})$, $7.49($ d. $J=8.37 \mathrm{~Hz}, 2 \mathrm{H}) .7 .55(\mathrm{~d}, J=8.08 \mathrm{~Hz} .2 \mathrm{H}), 7.60($ d, $J=8.31 \mathrm{~Hz} .2 \mathrm{H}$ ). 7.65 (d. $J=8.31 \mathrm{~Hz} .2 \mathrm{H}), 7.66$ (d. $J=8.60$ Hz. $2 \mathrm{H}), 7.69($ d. $J=8.60 \mathrm{~Hz}, 2 \mathrm{H}) ; \mathrm{HRMS}$ (EI, $70 \mathrm{eV}$ ) calcd for $\mathrm{C}_{31} \mathrm{H}_{30}\left(\mathrm{M}^{+}\right) 402.2348$. found 402.2371 .

(E)-4-[2-(t-tert-Butylphenyl)vinyl]-t"-tert-butyl-[1,1';4', $\mathbf{1}$ "Iterphenyl (8i) was prepared by the reaction of $\mathbf{6 c}(53.9$ mg. $0.100 \mathrm{mmol})$ with $7 \mathrm{c}(2.0 \mathrm{M}$ in Et_o, $0.15 \mathrm{~mL} .0 .30$ mmol $+0.10 \mathrm{~mL}, 0.20 \mathrm{mmol}$ ) in the presence of $\mathrm{dppfNiCl}$. The crude compound was purified by column chromatography $\left(\mathrm{CH}_{2} \mathrm{Cl}_{3}\right)$ to give $8 \mathbf{8}(32.9 \mathrm{mg} .0 .0740 \mathrm{mmol} .74 \%)$ as a white solid: $\mathrm{mp} 307-310^{\circ} \mathrm{C}$ (uncorrected): TLC $R_{f} 0.62$ $\left(\mathrm{CH}_{2} \mathrm{Cl}_{2}:\right.$-hexane $\left.=1: 1\right):{ }^{1} \mathrm{H}$ NMR $\left(500 \mathrm{MHz}, \mathrm{CDCl}_{2}\right) \delta$ $1.35(\mathrm{~s}, 9 \mathrm{H}) .1 .38(\mathrm{~s}, 9 \mathrm{H}), 7.12(\mathrm{~d} . J=16.34 \mathrm{~Hz}, 1 \mathrm{H}), 7.16$ (d. $J=16.34 \mathrm{~Hz}, 1 \mathrm{H}) .7 .40($ d. $J=8.41 \mathrm{~Hz}, 2 \mathrm{H}) .7 .49$ (d. $J=$ $8.41 \mathrm{~Hz}, 2 \mathrm{H}$ ). 7.49 (d. $J=8.45 \mathrm{~Hz}, 2 \mathrm{H}) .7 .60$ (d. $J=8.45 \mathrm{~Hz}$. $2 \mathrm{H}) .7 .60(\mathrm{~d} . J=8.40 \mathrm{~Hz}, 2 \mathrm{H}) .7 .65(\mathrm{~d} . J=8.40 \mathrm{~Hz}, 2 \mathrm{H})$. 7.67 (d. $J=8.43 \mathrm{~Hz} .2 \mathrm{H}$ ). 7.70 (d. $J=8.43 \mathrm{~Hz} .2 \mathrm{H}$ ): HRMS (EI. $70 \mathrm{eV}$ ) calcd for $\mathrm{C}_{34} \mathrm{H}_{36}\left(\mathrm{M}^{-}\right)$444.2817. found 444.2829 .

(E)-+-[2-(2-Methylbiphenyl-3-yl)vinyl]-t"-methy]-[1,1'; $4^{\prime}, 1$ "Iterphenyl (8j) was prepared by the reaction of $6 \mathbf{d}$ $(57.3 \mathrm{mg} .0 .100 \mathrm{mmol})$ with $7 \mathrm{~b}\left(1.0 \mathrm{M}\right.$ in $\mathrm{Et}_{2} \mathrm{O} .0 .30 \mathrm{~mL}$, $0.30 \mathrm{mmol}+0.20 \mathrm{~mL}, 0.20 \mathrm{mmol}$ ) in the presence of dppfNiCls. The crude compound was purified by column chromatography $\left(\mathrm{CH}_{2} \mathrm{Cl}_{2}\right)$ to give $8 \mathbf{j}(23.2 \mathrm{mg} .53 \%)$ as a pale yellow solid: mp $307.310^{\circ} \mathrm{C}$ (uncorrected); ${ }^{1} \mathrm{H}$ NMR $\left(500 \mathrm{MHz}, \mathrm{CDCl}_{3}\right) \delta 2.33$ (s, 3H). $2.4 \mathrm{l}$ (s. $\left.3 \mathrm{H}\right) .7 .07$ (d. $J=$ $16.20 \mathrm{~Hz} . \mathrm{lH}) .7 .19(\mathrm{~d}, J=7.50 \mathrm{~Hz}, 1 \mathrm{H}), 7.28($ d. $J=8.09$ Hz. 2H), 7.28 (t. $J=7.51 \mathrm{~Hz}, 1 \mathrm{H}$ ), 7.33 (dd. $J=8.24,1.44$ Hz. $2 \mathrm{H}$ ). 7.36 (t. $J=7.42 \mathrm{~Hz}, 1 \mathrm{H}) .7 .43(\mathrm{t} . J=7.57 \mathrm{~Hz}, 2 \mathrm{H}$ ), 7.48 (d. $J=16.20 \mathrm{~Hz} .1 \mathrm{H}) .7 .55$ (d. $J=8.09 \mathrm{~Hz} .2 \mathrm{H}$ ). 7.63 
(d. $J=8.20 \mathrm{~Hz}, 1 \mathrm{H}), 7.63$ (d. $J=8.52 \mathrm{~Hz} .2 \mathrm{H}) .7 .67$ (d, $J=$ $8.52 \mathrm{~Hz}, 2 \mathrm{H}), 7.67$ (d. $J=8.69 \mathrm{~Hz}, 2 \mathrm{H}), 7.70(\mathrm{~d}, J=8.69 \mathrm{~Hz}$. $2 \mathrm{H}$ ): HRMS (EI, $70 \mathrm{eV}$ ) calcd for $\mathrm{C}_{34} \mathrm{H}_{2}\left(\mathrm{M}^{+}\right) 436.219 \mathrm{l}$. found 436.2189 .

(E)-4-Methoxy-4"'-methyl-3-styryl-[1,1';4',1"]terpheny] (8k) was prepared by the reaction of $6 \mathrm{e}(51.3 \mathrm{mg}, 0.100$ mnol) with $7 \mathrm{~b}\left(1.0 \mathrm{M}\right.$ in $\mathrm{Et}_{2} \mathrm{O} .0 .30 \mathrm{~mL}, 0.30 \mathrm{nmmol}+0.20$ $\mathrm{mL} .0 .20 \mathrm{~mm}$ ol) in the presence of dppf $\mathrm{NiCl}_{2}$. The crude compound was purified by column chromatography $\left(\mathrm{CH}_{2} \mathrm{Cl}_{2}\right)$ to give $8 \mathbf{k}(24.1 \mathrm{mg}, 64 \%)$ as a pale yellow solid: $\mathrm{mp} 21 \mathrm{l}$ $214{ }^{\circ} \mathrm{C}$ (uncorrected); ${ }^{1} \mathrm{H}$ NMR $\left(500 \mathrm{MHz} . \mathrm{CDCl}_{3}\right) \delta 2.41$ (s. $3 \mathrm{H}), 3.95(\mathrm{~s}, 3 \mathrm{H}), 6.99(\mathrm{~d}, J=8.51 \mathrm{~Hz}, 1 \mathrm{H}), 7.20(\mathrm{~d}, J=$ $16.47 \mathrm{~Hz}, 1 \mathrm{H}), 7.24-7.28$ (n. $3 \mathrm{H}) .7 .36(\mathrm{t} . J=7.62 \mathrm{~Hz} .2 \mathrm{H}$ ). 7.52 (dd, $J=8.5 \mathrm{l}, 2.25 \mathrm{~Hz} . \mathrm{IH}), 7.53$ (d. $J=16.47 \mathrm{~Hz} . \mathrm{IH})$. 7.55 (d. $J=7.91 \mathrm{~Hz} .2 \mathrm{H}$ ). 7.57 (d. $J=7.13 \mathrm{~Hz}, 2 \mathrm{H}$ ). 7.67 (s. $4 \mathrm{H}), 7.86(\mathrm{~d} . J=2.25 \mathrm{~Hz}, \mathrm{IH}){ }^{13} \mathrm{C}$ NMR $\left(125 \mathrm{MHz} . \mathrm{CDCl}_{3}\right)$ $\delta 21.1,55.8,111.3,123.5,125.1,126.6(\times 2), 126.8,126.9$ $(\times 2), 127.1(\times 2) .127 .2 .127 .3(\times 2), 127.5,128.6(\times 2) .129 .5$ $(\times 2), 129.5,133.4 .137 .1,137.9 .137 .9,137.9 .139 .6 .156 .6$ : HRMS (EI, $70 \mathrm{eV}$ ) calcd for $\mathrm{C}_{2 \S \mathrm{H}_{2} 4} \mathrm{O}\left(\mathrm{M}^{+}\right) 376.1827$. found 376.1815 .

(E)-4-Methoxy-4"--methyl-3-[2-(4-tolyl)xiny]]-[1,1';4',1']terphenyl (81) was prepared by the reaction of $6 f(52.7 \mathrm{mg}$. $0.100 \mathrm{mmol}$ ) with $7 \mathrm{~b}(1.0 \mathrm{M}$ in Et $\mathrm{O}, 0.30 \mathrm{~mL} .0 .30 \mathrm{mmol}+$ $0.20 \mathrm{~mL} .0 .20 \mathrm{mumol}$ ) in the presence of dppfNiCls. The crude compound was purified by columu cluromatography $\left(\mathrm{CH}_{2} \mathrm{Cl}_{2}\right)$ to give $8 \mathbf{I}(28.1 \mathrm{mg} .72 \%)$ as a pale yellow solid: mp 266-268 ${ }^{\circ} \mathrm{C}$ (uncorrected); ${ }^{1} \mathrm{H}$ NMR (500 $\mathrm{MHz}, \mathrm{CDCl}_{3}$ ) $\delta 2.36$ (s. $3 \mathrm{H}$ ). 2.41 (s. $3 \mathrm{H}$ ), 3.94 (s. $3 \mathrm{H}$ ), 6.99 (d. $J=8.52$ $\mathrm{Hz}, \mathrm{IH}$ ). 7.17 (d. $J=8.00 \mathrm{~Hz}, 2 \mathrm{H}$ ). 7.18 (d, $J=16.48 \mathrm{~Hz}$. lH), 7.27 (d. $J=8.07 \mathrm{~Hz} .2 \mathrm{H}), 7.46$ (d. $J=8.00 \mathrm{~Hz}, 2 \mathrm{H}$ ). $7.48(\mathrm{~d} . J=16.48 \mathrm{~Hz}, \mathrm{IH}) .7 .50(\mathrm{dd}, J=8.52 .2 .26 \mathrm{~Hz} . \mathrm{lH})$. 7.55 (d. $J=8.07 \mathrm{~Hz} .2 \mathrm{H}), 7.66(\mathrm{~s}, 4 \mathrm{H}), 7.85$ (d. $J=2.26 \mathrm{~Hz}$. 1H): ${ }^{13} \mathrm{C}$ NMR (125 MHz, $\left.\mathrm{CDCl}_{3}\right) \delta 21.1,21.3 .55 .7,111.3$. 122.4. 125.0. 126.6 (×2), 126.9, $126.9(\times 2) .127 .0 .127 .1$ $(\times 2), 127.3(\times 2), 129.3(\times 2), 129.5,129.5(\times 2), 133.4,135.1$. 137.1. 137.3, 137.9. 139.6. 139.6, 156.5: HRMS (EI, 70 eV) calcd for $\mathrm{C}_{20} \mathrm{H}_{36} \mathrm{O}\left(\mathrm{M}^{-}\right) 390.1984$, found 390.2012 .

(E)-4-Methoxy-4"-methyl-3-[2-(4-tert-butyl]phenyl)vinyl]-[1,1';4',1"]terphenyl (8m) was prepared by the reaction of $6 \mathrm{~g}(56.9 \mathrm{mg} .0 .100 \mathrm{mmol})$ with $7 \mathrm{~b}\left(2.0 \mathrm{M}\right.$ in $\mathrm{Et}_{2} \mathrm{O} .0 .15$ $\mathrm{mL} .0 .30 \mathrm{mmol}+0.10 \mathrm{~mL} .0 .20 \mathrm{mmol}$ ) in the presence of dppfNiCls. The crude compound was purified by colunn chromatography $\left(\mathrm{CH}_{2} \mathrm{Cl}_{2}\right)$ to give $8 \mathrm{~m}(32.9 \mathrm{mig} .76 \%)$ as a pale yellow solid: mp $239-241{ }^{\circ} \mathrm{C}$ (uncorrected): ${ }^{1} \mathrm{H}$ NMR $\left(500 \mathrm{MHz}, \mathrm{CDCl}_{3}\right) \delta \mathrm{l} .34$ (s. 9H), $2.4 \mathrm{l}$ (s. $\left.3 \mathrm{H}\right), 3.93$ (s, 3H). 6.98 (d. $J=8.54 \mathrm{~Hz}, 1 \mathrm{H}), 7.19(\mathrm{~d}, J=16.45 \mathrm{~Hz}, 1 \mathrm{H}), 7.27$ (d. $J=8.08 \mathrm{~Hz}, 2 \mathrm{H}) .7 .39$ (d. $J=8.40 \mathrm{~Hz}, 2 \mathrm{H}) .7 .49$ (d. $J=$ $16.45 \mathrm{~Hz}, 1 \mathrm{H}$ ). $7.50(\mathrm{~d}, J=8.40 \mathrm{~Hz}, 2 \mathrm{H}) .7 .50$ (dd $J=8.54$. $2.25 \mathrm{~Hz}, 1 \mathrm{H}$ ) .7 .55 (d. $J=8.08 \mathrm{~Hz}, 2 \mathrm{H}) .7 .67$ (s. $4 \mathrm{H}) .7 .85$ (d. $J=2.25 \mathrm{~Hz}, 1 \mathrm{H}):{ }^{13} \mathrm{C} \mathrm{NMR}\left(125 \mathrm{MHz} . \mathrm{CDCl}_{3}\right) \delta 21.1$. 31.3 (×3). 34.6, 55.8, 111.3. 122.7. 125.0. 125.5(×2), 126.4 $(\times 2), 126.9(\times 2), 127.0,127.0,127.1(\times 2), 127.3(\times 2), 129.3$. $129.5(\times 2) .133 .4,135.2,137.1 .137 .9 .139 .6,139.6,150.6$. 156.5: HRMS (EI, 70 eV) calcd for $\mathrm{C}_{3 \mathrm{3}} \mathrm{H}_{33} \mathrm{O}\left(\mathrm{M}^{+}\right) 432.2453$. found 432.2461 .
Acknowledgement. This research was supported by the Chung-Ang University Research grants in 2005.

\section{References}

1. (a) Walter. G: Liebl. R.: Von Angerer. E. Bioong Med. Chent. 2004. 14. 4659. (b) Chávez. D.: Chai. H. B.: Chagwedera. T. E.: Gao. Q: Fartisworth. N. R.: Cordell. G. A.: Pezzuto. I. M: Kinghom, A. D. Tetrahedron Lett 2001. 42, 3685. (c) Jang, M: Cai. L.: Udeani. G. O.: Slowing, K. V: Thomas, C. F.: Beecher. C. W. W.: Fong. H. H.: Fansworth. N. R.: Kinghorn. A. D.: Mehta. R. G.: Moon. R. C.: Pezzuto. J. M. Science 1997. 275. 218.

2. Ducrot. P.-H.: Kollmann. A.: Bala. A. E.: Majira. A.: Kerhoas. L.: Delorme. R.: Eitnhort1. J. Tetrahedron Lett. 1998. 39. 9655

3. Iliva, I.: Ali. Z; Tanaka. T.: Inuma, M; Furusawa, M; Nakaya. K.i.: Murata, J.: Damaedi, D; Matsuura, N.; Ubukata, M. Phvtochemisty 2003, 62,601.

4. Dai. J. R.: Hallock. Y. F.: Cardellina. II. T. H.: Boyd. M. R. J. Nat. Prod. 1998. 61.351

5. Huang. K.-S.: Li. R.-L.: Wang. Y.-H.: Lin. M. Planta. Med. 2001. 67. 61 .

6. (a) Savouret. J. F; Quesne, M. Bioned Phamacother 2002. 56. 84. (b) Gusman, J.: Malonne, H.: Atassi, G. Carcinogenesis 2001. 22. 1111 . (c) Frémont. L. Lifé Sci. 20(00. 66.663.

7. Momotake A.: Arai. T. J. Photochent Photobiol A: Photochent Reviews 2004. 5. 1

8. (a) Imai. M. Arai. T. Tetrahedron Lett. 2002. 43. 5265 . (b) Meier H. Angew. Chem. Iht Ed. 1992. 31, 1399. (c) Waldeck, D. H Chem. Rev 1991. 91.415.

9. (a) Feringa. L.: Jager. W. F.: de Lange. B. Tetrohedron 1993. 49. 8267. (b) Emmelius. M.: Pawlowski. G.: Vollmann. H. W. Angew: Chent Int. Ed. 1989. 28. 1445

10. (a) Romaniello, P; Lelj, F. J. Fhorine Chem. 2004. 125, 145. (b) Papagni, A.; Maiorana, S.: Del Buttero. P.: Perdicchia. D.: Cariati. F.: Cariati, E: Marcolli. W. Em: J. Org. Chem 2002, 8. 1380. (c) Lipiñski. T.: Bartkowiak. W. Chem. Phs 1999. 245. 263. (d) Kiml. H. K.: Moonl. I. K.: Lee. H.-J: Han. S.G.: Won. Y. H. Polyner 1998. 39. 1719. (e) Marder. S. R.: Kippelen. B.: Ten. A. K.-Y.; Peyghambarian. N. Nature 1997, 388. 845. (f) Ashwell, G. J.; Jefferies, G; Hamilton. D. G.; Lynch, D. E.: Roberts. M. P. S.; Bahra. G. S.: Brown, C. R. Nature 1995. 375. 385 . (g) Chen, C.-T: Marder. S. R.: Cheng. L.-T. J. Chem. Soc. Chem. Conmmin. 1994. 259.

11. Burroughes. T. H.: Bradley. D. D. C.: Brown. A. R.: Marks. R. N.: Medray, K.; Friend, R. H.; Burns, P. L.: Holmes, A. B. Natwe $1990.3+7,539$

12. (a) Jin, Y.: Kim. J.: Park, S. H.: Kim. H.; Lee, K: Sulh, H. Bull. Korem Chent. Soc. 2005. 26. 1807. (b) Kraft. A.: Grimsdale. A C.: Holmes. A. B. Angew: Chent Int Ed 1998. 37. 402. (c) Gustafsson. G.: Cao. Y.: Treacy. G. M.: Klavetter. F.: Colaneri. N.: Heeger. A. J. Fatwe 1992, 357, 477. (d) Burns. P. L.: Holmes, A. B; Kraft. A.; Bradley. D. D. C.: Brown, A. R.: Friend, R. H. Nature 1992, 356.47.

13. (a) Hohnholz. D.: Schweikart. K.-H.: Subramaniant. L. R.: Wedel. A.: Wischert. W.: Hanack. M. Simth. Met 2000. 110. 141. (b) Martin. R. E.: Diederich. F. Angew. Chent. Int. Ed 1999. 38. 1350. (c) Hohloch. M:; Segura, J. L.; Doettinger. S. E.: Hohnholz, D.; Steinhuber, E.; Spreitzer. H.: Hanack, M. Symh . Het. 1997.84. 319. (d) Döttinger, S. E.; Hohloch, M.: Hohnholz, D; Segura. J. L.: Steinhuber. E.: Hanack. M. Smth. Met. 1997. 8t. 267.

14. Mooney III. W. F.: Brown. P. E.: Russell. J. C.: Costa. S. B.: Pedersen. L. G.: Whitten. D. G. J. Am. Chent Soc. 1984. 106. 5659.

15. (a) Um. S.-I.; Lee, J.-K.: Kang. Y:; Baek. D.-J. Dyes Pigments 2005. 64, 93. (b) Sengupta. S. Tetrahedron Lett 2003, H, 307. (c) Sigalor. M.: Betr-Asuly. A.: Shapiro. L:: Ellem1. A.: Khodorkorshy. 
V. Tetrahedron Lett. 2000 . H. 8573

16. (a) Xu. Q.: Chen. H. Z.: Wang. M. Mater Chent Phis. 2004. 87. 446. (b) Zhan. C.: Cheng. Z.: Zheng. J.: Zhang. W.: Xi. Y: Qin. J. J. Appl Polym. Sci. 2002, 85, 2718. (c) Yang, J: Lin, H: Zheng. M.: Bai, F. Hacromol Chem. Plns. 2001. 202, 2287.

17. (a) Markham, J. P. J.: Nandas. E. B.: Anthopoulos. T. D.: Samulel, I. D. W.: Richards. G. J.: Burn. P. L. Appl. Plvs. Lett. 2004. 85. 1463. (b) Sengupta. S.: Muluri. S. Tetrahedron Lett. 2004. 15. 2895. (c) Ma. D.: Hu. Y.: Zhang. Y.: Wang. L.: Jing. X.: Wang. F.: Lupton, I. M.: Samuel, I. D. W.: Lo, S.-C : Burn. P. L. Symhetic Wetals 2003, 137, 1125. (d) Lupton, J. M: Samuel. I. D. W: Frampton, M. I.: Beavington, R: Burn. P. L. Ad: Funct. Mater. 2001. II. 287. (e) Behnisch. B.: Hanack. M. Sinthetic Metals 2001. I21. 1661. (f) Lupton. J. M.: Hemingway. L. R.: Samuel. I. D. W.: Burn. P. L. J. Mater: Chemt 2000. 10. 867. (g) Halim. M: Pillow: J. N. G.; Samuel. I. D. W.: Burn, P. L. Adv Hater 1999. 11 . 371 .

18. Becker. K. B. Symthesis $1983,341$.

19. (a) Eichen. Y: Nakhmanovich. G.: Gorelik. V: Epshtein. O. Poplawski. J. M.: Ehrenfreund. E. J. Am. Chen. Soc. 1998. 120. 10463. (b) Orsini. F.: Pelizzoni. F.: Bellini. B.: Miglierini. G. Carbolydrate Research 1997, 301, 95. (c) Rao, V. P: Tell. A. K.-Y: Wong. K. Y: Drost. K. J. Tetrahedron Leth. 1993, 34. 1747

20. (a) Diez-Barra. E.: Garcia-Martinez, J. C.: Rodriguez-López. J. Tetrohedron Lett. 1999, 10.8181. (b) Wang. M.: Jin. Y.: Ho. C.-T. J. Agric. Food Chem 1999. 47. 3974. (c) Meier. H.: Dullweber. U. Tetrahedron Lett. 1996, 37, 1191

21. (a) Sengupta, S.: Sadhlkhan. S. K.: Singh, R. S.: Pal. N Tetrahedron Lett. 2002, t3. 1117. (b) Guiso. M.: Marra, C.:
Farita. A. Tetratedron Lett. 2002. 43. 597. (c) Bazan. G. C.: Oldham. Jr. W. T.: Lachicotte. R. J.: Tretiak. S.: Chernyak. V: Mukamel. S. J. An Chem. Soc. 1998. 120.9188. (d) Belfield. K D.: Chinna, C.: Schafer. K. J. Tetahedron Lett. 1997, 38.6131.

22. (a) Jeffery. T.: Ferber. B. Tetrahedron Lett. 2003. H, 193 . (b) Kiji. J.; Okano. T:; Ooue, A. J. Hol Catal A: Chem 1999, 147. 3. (c) Spencer. A.J. Onganomet. Chent 1983. 247. 117.

23. (a) Tietze. L. F.: Kettschaul. G.: Heuschert. U.: Nordmann. G Chent Ent J. 2001. 7. 368. (b) Tietze. L. F.: Nordmann. G. Smlet 2001.337.

24. Maddux. T; Li. W; Yu. L. J. Am. Chem Soc, 1997, 119.844.

25. Solladié, G:; Pasturel-Jacope. Y: Maignan, J. Tetrahedron 2003. 59.3315

26. (a) Liu. K. H.: Masuda. A.: Yamazaki. Y. Tetrahedron Lett. 1975. 3007. (b) Treves. G. R. J. An. Chem. Soc. 1948. 70. 875

27. Hauser. C. R.: Brasen, W. R.: Skell. P. S.: Kantor, S. W.: Brodhag. A. E. J. Am. Chem. Soc. 1956, 78, 1653 .

28. (a) MoMurn; J. E; Fleming. M. P. J. Am Chem. Soc. 1974. 96. 4708 . (b) Stantield. T. A.: Revnolds. Jr. L. B. J. Ant Chent. Soc. 1952. 7f. 2878 .

29. (a) Cho. C.-H.: Sut1. M.: Seo. Y.-S.: Kim. C.-B.: Park. K. J. Org Chem. 2005, 70, 1482. (b) Cho. C.H.: Sun. M.: Park, K. Bull. Korean Chem. Soc. 2005, 26, 1410, (c) Cho, C.-H.: Kim, I.-S.: Park. K. Tetrahedron 2004, 70. 4589. (d) Cho. C.-H.: Yun, H.-S. Park. K. J. Ong. Chem. 2003. 68.3017.

30. Cho. C.-H.: Kim. C.-B.: Sun1. M.: Park. K. Bull. Korean Chem Soc. 2003.24 .1632

31. (a) Wadsworth, D. H.: Schupp. III, O. E.: Seus. E. J.; Ford, Jr., J. A. J. Org. Chem. 1965, 30. 680. (b) Wadsworth. Jr. W. S. Emmons. W. D. J. Am. Chem. Soc. 1961. 83, 1733. 